\title{
Double-Cap Differential Codebook Structure for MU MISO Systems in Correlated Channels
}

\author{
Jawad Mirza, Student Member, IEEE, Peter J. Smith, Senior Member, IEEE, Mansoor Shafi, Fellow, IEEE \\ Pawel A. Dmochowski, Senior Member, IEEE, Abdulla Firag, Member, IEEE, and Apostolos Papathanassiou
}

\begin{abstract}
We propose a double-cap differential codebook (DCB) design for multiuser (MU) multiple-input-single-output systems. The MU transmission uses signal-to-leakage-and-noise ratio (SLNR) precoding. The double-cap DCB is designed for temporally/spatially correlated channels and is based on a combined spherical and polar cap structure. We consider an inner, spherical cap and an outer, polar cap structures with different radii. The design allows us to systematically alter the radii of the caps over the feedback time depending on the previously selected codeword. The proposed double-cap DCB does not require knowledge of temporal or spatial channel correlation at the base-station (BS), thus removing the overhead of additional feedback from the user. Simulation results show the effectiveness of the proposed double-cap DCB over a single polar cap DCB in terms of sum capacity gain and quantization errors.
\end{abstract}

Index Terms-Limited feedback MISO systems, SLNR precoding, differential codebook (DCB), muti-user MIMO, spatiotemporal correlation.

\section{INTRODUCTION}

Research into limited feedback multiple-input multipleoutput (MIMO) systems began more than a decade ago. The early work was dominated by codebook designs for independent and identically distributed (i.i.d.) Rayleigh fading channels, e.g. [1], [2]. Later, the focus of the research shifted towards correlated channels where channel entries are either spatially correlated or temporally correlated or both. For spatially correlated MIMO channels, the key idea is to maneuver the codewords of the Grassmannian codebook [1] in the direction of the channel using the transmit correlation matrix [3], [4]. These codebooks, known as spherical codebooks, outperform the Grassmannian line packing (GLP) codebook [1] in spatially correlated channels. However, in this scheme, the knowledge of the spatial correlation must be shared with the BS. Similarly, for temporally correlated MIMO channels, differential limited feedback schemes are proposed in [5]-[8] where the codebook radius changes with time depending on the temporal correlation in the channel. This adaptation has been shown to track the channel successfully. The adaptive or time varying radius of the cap is referred to as adaptive scaling in this paper. Such codebooks are also known as differential codebooks (DCBs). Differential codebooks that exploit temporal correlation in MIMO channels are also studied in [9][11]. The drawback of many DCBs is the requirement of a periodical reset to avoid error propagation.

The main motivation of this paper is to design a DCB structure for correlated MISO channels that efficiently quantizes the channel direction information (CDI) and performs adaptive scaling to keep track of the channel without the requirement of additional feedback or reset. To achieve these goals, we propose a double-cap DCB where two different cap structures are positioned on a Grassmannian manifold with different radii, centered around the same location. We consider an inner spherical cap and an outer polar cap. The codewords on the circumference of the outer cap are used to quantize the channel vector and to enable rapid or large scale tracking of the channel. On the other hand, the spherical cap codewords are distributed inside the cap, hence reducing quantization errors once the DCB closely follows the channel. As will be shown, splitting the codewords into two distinct subsets allows us to signal the need for radius adaptation without additional feedback overhead. The main contributions of this paper are:

- We propose a double-cap DCB design based on an inner spherical and outer polar cap structure for MU MISO systems under correlated channels modeled using the WINNER II channel model [12].

- We present a blind adaptive scaling technique where cap radii are systematically adjusted after each feedback interval.

- Based on an exact calculation of the average chordal distance between an i.i.d. Rayleigh fading channel and the selected random vector quantization (RVQ) codeword, we derive the initial radii for the caps.

Compared to the previous literature, this work demonstrates improved performance compared to [5] and [6] without the need for reset and without any extra feedback such as codebook switching or correlation information [11].

Notation: We use $\mathbf{A}^{H}, \mathbf{A}^{T}, \mathbf{A}^{-1}$, and $\mathbf{A}^{\perp}$ to denote the conjugate transpose, the transpose, the inverse and the null space of the matrix $\mathbf{A}$, respectively. $\|\cdot\|$ and $|\cdot|$ denote vector and scalar norms, respectively. $\mathbb{E}[\cdot]$ denotes expectation.

\section{System Model}

Consider a MU MISO system with a single BS serving $K$ single antenna users. The number of transmit antennas at the BS is $M$ and we assume $K=M$. On the downlink, the received signal at user $k$ for the transmitted data symbol, $s_{k}$, is given by

$$
r_{k}=\left(\mathbf{h}_{k} \mathbf{w}_{k}\right) s_{k}+\sum_{j=1, j \neq k}^{K}\left(\mathbf{h}_{k} \mathbf{w}_{j}\right) s_{j}+z_{k},
$$

where $\mathbf{h}_{k}$ denotes the $1 \times M$ channel vector between BS and user $k$ and follows the WINNER II channel model. The precoding vector of size $M \times 1$ for the $k^{\text {th }}$ user is denoted by $\mathbf{w}_{k}$ and $z_{k}$ is an additive white Gaussian noise (AWGN) 
term at the $k^{\text {th }}$ user, such that $z_{k} \sim \mathcal{C N}\left(0, \sigma_{k}^{2}\right)$. The signalto-noise ratio (SNR) for systems without precoding is given by $1 / \sigma_{k}^{2}$ [13]. We assume equal noise variance and average power for all users. The data symbols and precoding vector are normalized such that $\mathbb{E}\left[\left|s_{k}\right|^{2}\right]=\left\|\mathbf{w}_{k}\right\|^{2}=1, \forall k$. If the interference is treated as Gaussian noise, then the sum rate of the MU MISO system becomes

$$
\mathbf{R}_{\text {sum }}=\sum_{k=1}^{K} \log _{2}\left(1+\frac{\left|\mathbf{h}_{k} \mathbf{w}_{k}\right|^{2}}{\sigma_{k}^{2}+\sum_{j=1, j \neq k}^{K}\left|\mathbf{h}_{k} \mathbf{w}_{j}\right|^{2}}\right)
$$

Each user maintains a codebook consisting of $2^{B}$ possible CDI entries (or codewords), where $B$ is the number of feedback bits. We denote the codebook at user $k$ by $\mathbf{F}_{k}=$ $\left[\mathbf{f}_{k}^{(1)}, \mathbf{f}_{k}^{(2)}, \ldots, \mathbf{f}_{k}^{\left(2^{B}\right)}\right]$ where each codeword is a column vector of size $M \times 1$. The selected codeword, determined by the minimum chordal distance, is given by

$$
\overline{\mathbf{f}}_{k}=\underset{1 \leq i \leq 2^{B}}{\operatorname{argmin}} \sqrt{1-\left|\overline{\mathbf{h}}_{k} \mathbf{f}_{k}^{(i)}\right|^{2}}
$$

where, $\overline{\mathbf{h}}_{k}=\mathbf{h}_{k} /\left\|\mathbf{h}_{k}\right\|$ is the true channel direction. We assume that the BS has perfect knowledge of the channel quality indicator $^{1}$ (CQI) for each user, where CQI $=\left\|\mathbf{h}_{k}\right\|$. The BS finds the quantized channel, $\hat{\mathbf{f}}_{k}$, using the selected codeword $\overline{\mathbf{f}}_{k}$ and the CQI such that $\hat{\mathbf{f}}_{k}=\left\|\mathbf{h}_{k}\right\| \overline{\mathbf{f}}_{k}^{T}$.

We use the signal-to-leakage-and-noise ratio (SLNR) based precoding technique proposed in [13] for MU MIMO downlink communications. SLNR precoding has been shown to outperform zero-forcing (ZF) precoding in the low SNR regime by reducing noise inflation [13] and in statistical beamforming an SLNR type structure has been shown to be near-optimal [14]. In a limited feedback MISO system, the SLNR precoding vector, $\mathbf{w}_{k}$, for the $k^{\text {th }}$ user is the normalized version of

$$
\mathbf{w}_{k}=\left(\sigma_{k}^{2} \mathbf{I}+\mathbf{H}_{k}^{H} \mathbf{H}_{k}\right)^{-1} \hat{\mathbf{f}}_{k}^{H},
$$

where $\mathbf{H}_{k}$ is an extended channel matrix of size $(K-1) \times M$ for the $k^{\text {th }}$ user constructed from the quantized channels of all users, $\mathbf{H}_{k}=\left[\hat{\mathbf{f}}_{1} \cdots \hat{\mathbf{f}}_{k-1} \hat{\mathbf{f}}_{k+1} \cdots \hat{\mathbf{f}}_{K}\right]^{T}$.

\section{REVIEW OF SPHERICAL CAP AND POLAR CAP CODEBOOK DESIGN}

In this section, we briefly explain the structures of the spherical cap [4] and polar cap [6]. The DCB proposed in this paper combines both caps to design a double-cap DCB. In this section, we drop the user index, $k$, for clarity.

\section{A. Polar cap structure}

The polar cap DCB consists of a basis vector of size $M \times 1$ given by $\mathbf{c}=[1,0, \cdots, 0]^{T}$ that is also the center of the polar-cap. The basis vector is not a codeword and all $2^{B}$ codewords are located on the circumference of the polar-cap. In order to generate these $2^{B}$ codewords, a Grassmannian line packing (GLP) codebook [1], denoted by $G(M, 1)$, is generated where each codeword is of size $(M-1) \times 1$, given

\footnotetext{
${ }^{1}$ As in [5], [6], the main objective of this paper is to study the effect of CDI quantization on the performance of the MU MISO system. Hence CQI quantization is not considered in this paper.
}

by $\tilde{\mathbf{F}}=\left[\tilde{\mathbf{f}}_{1}, \cdots, \tilde{\mathbf{f}}_{2^{B}}\right]$. The next step is to set an appropriate distance, $\alpha$, also referred to as a scaling parameter in this paper, that defines the distance between the basis vector $\mathbf{c}$ and the $2^{B}$ codewords. Once $\alpha$ is set, then the polar cap DCB, $\mathbf{W}_{\text {polar }}$, of size $2^{B}$ is generated by [6]

$$
\mathbf{W}_{\text {polar }}=\left\{\left[\begin{array}{c}
\sqrt{1-\alpha^{2}} \\
\alpha \tilde{\mathbf{f}}_{1}
\end{array}\right], \cdots,\left[\begin{array}{c}
\sqrt{1-\alpha^{2}} \\
\alpha \tilde{\mathbf{f}}_{2^{B}}
\end{array}\right]\right\} .
$$

\section{B. Spherical cap structure}

The spherical cap is also centered on $\mathbf{c}=[1,0, \cdots, 0]^{T}$. Like the polar cap, it does not include $\mathbf{c}$ as one of the codewords. We first generate a spherical cap of size $2^{B}$ on $G(M, 1)$ with center $\mathbf{c}$ and radius $\gamma$, denoted by $\mathbb{O}(\mathbf{c}, \gamma)$. The spherical cap is defined as an open set $\mathbb{O}(\mathbf{c}, \gamma)=\{\mathbf{f} \in$ $\left.G(M, 1): \sqrt{1-\left|\mathbf{c}^{H} \mathbf{f}\right|^{2}}<\gamma\right\}$, where we set $\gamma=0.99$. Each codeword is scaled individually [4] to give the spherical cap DCB,

$\mathbf{W}_{\text {spherical }}=$
$\left\{\left[\begin{array}{c}\sqrt{1-\delta^{2}\left(1-r_{1,1}^{2}\right)} e^{j \theta_{1,1}} \\ \vdots \\ \delta r_{M, 1} e^{j \theta_{M, 1}}\end{array}\right], \cdots,\left[\begin{array}{c}\sqrt{1-\delta^{2}\left(1-r_{1,2^{B}}^{2}\right)} e^{j \theta_{1,2} B} \\ \vdots \\ \delta r_{M, 2} B \\ e^{j \theta_{M, 2} B}\end{array}\right]\right\}$,

where $r_{m, n} e^{j \theta_{m, n}}$ is the polar form of the $m^{\text {th }}$ entry of the $n^{\text {th }}$ GLP codeword and $\delta$ is a scaling parameter satisfying $0<\delta<\gamma$. Note that $\delta \times \gamma \approx \delta$, as $\gamma \approx 1$.

\section{PRoposed DOUBLE-CAP DCB}

We propose a double-cap DCB based on polar and spherical cap structures with different radii. Following the first feedback interval, where an RVQ codebook is used, the double-cap DCB is centered around the selected RVQ codeword. Intuitively, it would be appropriate to scale the codebook using knowledge of the quantization error due to the selected RVQ codeword. The initial radius, $\delta$, for the inner cap (spherical cap) is set to the average distance between the selected RVQ codeword, $\overline{\mathbf{f}}_{k}$, and the channel direction, $\overline{\mathbf{h}}_{k}$. The outer polar cap radius is set to the maximum quantization error limit, $\alpha=1$. Hence, for smaller than average errors we use the inner spherical cap for fine tracking of the channel. When the quantization error is larger than average, the outer polar cap codewords are used for quantizing and large scale tracking of the channel. Following the second feedback interval, the double-cap DCB radii follow the blind adaptive scaling technique discussed in Section IV-B. We can write the distance, $\delta$, as

$$
\delta=\mathbb{E}\left[\sqrt{1-\left|\overline{\mathbf{f}}_{k} \overline{\mathbf{h}}_{k}\right|^{2}}\right] .
$$

As shown in [15], the random variable $X=\sqrt{1-\left|\mathbf{f}_{k}^{(i)} \overline{\mathbf{h}}_{k}\right|^{2}}$ is beta distributed with parameters $2(M-1)$ and 1 . Let $y$ be the minimum of the $2^{B}$ random variables, $X$, then the probability density function (PDF) of $y$ can be written as

$$
f(y)=2^{B} f(x)(1-F(x))^{2^{B}-1}, \quad 0<y<1,
$$


where $f(x)$ and $F(x)$ are the PDF and cumulative distribution function $(\mathrm{CDF})$ of the independent $X$ variables, given by

$$
f(x)=2(M-1) x^{(2 M-3)}
$$

and

$$
F(x)=x^{2(M-1)} .
$$

Substituting (9) and (10) in (8), we have

$$
f(y)=2^{B+1}(M-1) y^{(2 M-3)}\left(1-y^{2(M-1)}\right)^{2^{B}-1} .
$$

Using $f(y)$, we can calculate the value of $\delta$ in (7) as

$$
\begin{gathered}
\delta=\int_{0}^{1} y f(y) d y \\
=2^{B+1}(M-1) \int_{0}^{1} y^{2(M-1)}\left(1-y^{2(M-1)}\right)^{2^{B}-1} d y \\
=2^{B} \beta\left(\frac{M-\frac{1}{2}}{M-1}, 2^{B}\right) .
\end{gathered}
$$

This distance gives the exact value as compared to [15] where a similar bound is derived for the squared chordal distance. We set the radius of the inner cap (spherical cap) to $\delta$ given in (14). After setting the outer and inner cap radii, the doublecap DCB structure is obtained by generating a spherical cap with radius $\delta$ and a polar cap with radius $\alpha$. This double-cap DCB is centered around the basis vector, $\mathbf{c}$, which is not a codeword. The double-cap DCB design is shown in Fig. 1. The number of codewords is evenly distributed on both inner

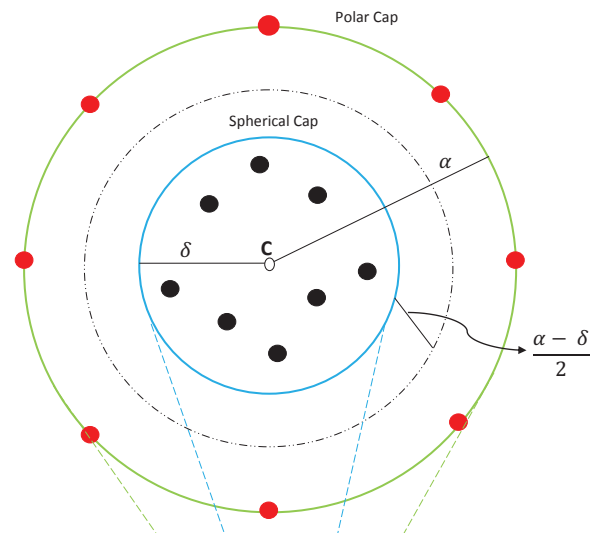

Fig. 1. The structure of the double-cap DCB based on polar/spherical caps. and outer caps i.e $2^{B} / 2$ codewords on each cap ${ }^{2}$.

\section{A. Feedback procedure}

The feedback procedure for the double-cap DCB is similar to the feedback rules in [6]:

- For the first feedback at time $t-1$, the user selects a codeword, $\overline{\mathbf{f}}_{k}[t-1]$, from the base codebook (e.g. GLP, RVQ, or 3GPP LTE) and sends its index to the BS.

- For the next feedback at time $t$, the basis vector, c, is rotated to the previously selected codeword, $\overline{\mathbf{f}}_{k}[t-1]$. The rotation matrix is given by

$$
\Theta_{\overline{\mathbf{f}}_{k}[t-1]}=\left[\begin{array}{ll}
\overline{\mathbf{f}}_{k}[t-1] & \overline{\mathbf{f}}_{k}[t-1]^{\perp}
\end{array}\right]\left[\begin{array}{ll}
\mathbf{c} & \mathbf{c}^{\perp}
\end{array}\right]^{H} .
$$

${ }^{2} \mathrm{~A}$ double-cap DCB can also be designed with unequal numbers of codewords on the two caps. Due to space constraints we only consider the equal distribution.
All the codewords in both caps are rotated by this rotation matrix such that new a double-cap DCB is created at both user and BS with the center at $\overline{\mathbf{f}}_{k}[t-1]$.

- The double-cap DCB process continues and adapts the radii of the caps blindly.

\section{B. Blind adaptive scaling}

The advantage of having two different radii in the codebook is that the codebook can be scaled up or scaled down depending on the previously selected codeword (i.e. without additional feedback). In order to track the channel over time, we need to maintain a distance between inner and outer caps. Therefore, we shrink and expand both the caps by $\frac{\alpha-\delta}{2}$. The double-cap DCB expansion and contraction is described below.

- If the previous selected codeword at time $t$ is from the outer polar cap then expand the double-cap DCB for the next feedback such that $\alpha[t+1]=\alpha[t]+(\alpha[t]-\delta[t]) / 2$ and $\delta[t+1]=\delta[t]+(\alpha[t]-\delta[t]) / 2$.

- If the previous selected codeword is from the inner spherical cap then contract the double-cap DCB such that $\delta[t+1]=\delta[t]-(\alpha[t]-\delta[t]) / 2$ and $\alpha[t+1]=$ $\alpha[t]-(\alpha[t]-\delta[t]) / 2$.

This method does not require the BS to have any information about spatial and temporal correlation in the channel.

\section{Simulation Results}

We perform Monte-Carlo simulations to evaluate the performance of the proposed double-cap DCB and compare it with the single polar cap DCB [6] (that outperforms [5]) over spatially/temporally correlated MISO channels. The BS has 4 antennas and is serving 4 users simultaneously. The base codebook is a 4 bit RVQ codebook. The carrier frequency is 2.5 GHz. The MU MISO channel is modeled using the WINNER II channel [12] with an urban macro (Uma) environment and non-line-of-sight (NLOS) propagation. The spacing between transmit antennas is $0.5 \lambda$. We assume a lossless feedback link with zero delay. It is also assumed that perfect CQI is available at the BS and all the users have the same speed with a $5 \mathrm{~ms}$ feedback interval. In addition to CDI quantization errors, we also analyze the impact of channel estimation errors at the user. The estimated channel is given by

$$
\tilde{\mathbf{h}}_{k}=\epsilon \mathbf{h}_{k}+\sqrt{1-\epsilon^{2}} \mathbf{g}_{k},
$$

where $\epsilon$ is a measure of the CSI accuracy, $0 \leq \epsilon \leq 1$ and $\mathrm{g}_{k} \sim \mathcal{C N}(0,1)$.

Figure 2 shows the sum-rate versus time results for the double-cap DCB at SNR $=10 \mathrm{~dB}$. The speed of the users is $v=1 \mathrm{~km} / \mathrm{h}$. The codewords are evenly distributed on both caps (i.e. 8 on the inner and 8 on the outer cap) in the double-cap DCB, The sum-rate gain achieved by the proposed double-cap DCB is significant and outperforms the single polar cap DCB. The codebook reset time for the single polar cap DCB is set to 9 feedback intervals [6]. It is important to note here that the double-cap DCB is using blind adaptive scaling, whereas in the case of the single polar cap DCB, the adaptive scaling assumes that the BS has perfect temporal correlation 


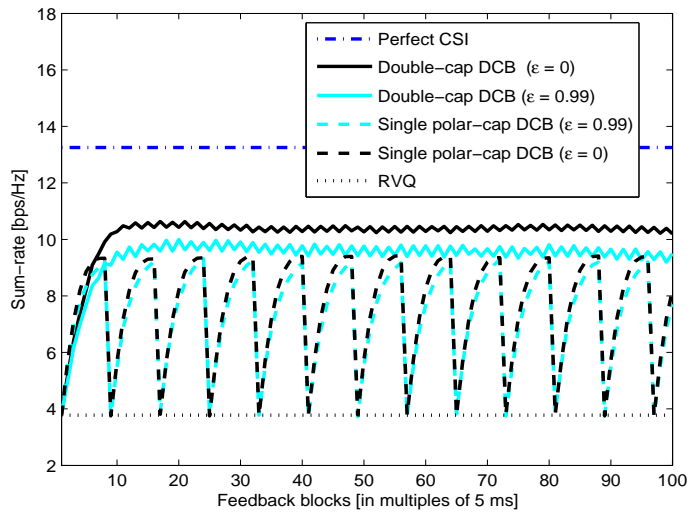

Fig. 2. Sum rate versus time at $\mathrm{SNR}=10 \mathrm{~dB}$ with $\mathrm{v}=1 \mathrm{~km} / \mathrm{h}$ and 4 bit feedback.

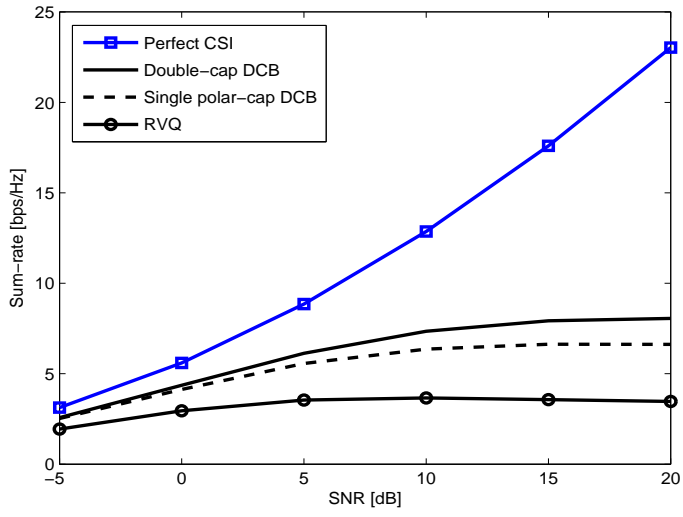

Fig. 3. Sum rate results against SNR with $v=3 \mathrm{~km} / \mathrm{h}$ and 4 bit feedback (100 feedback intervals and $\epsilon=0$ ).

information. The small channel estimation error, $\epsilon=0.99$, at the user degrades the quantization performance of the system, implying that DCB is sensitive to channel estimation errors. Sum-rate results are plotted against different SNR values in Fig. 3 for $v=3 \mathrm{~km} / \mathrm{h}$. The double-cap DCB gives superior sum-rate performance compared with single polar-cap DCB and RVQ codebooks with 4 bit feedback. Figure 4 shows a $\mathrm{CDF}$ plot for the quantization errors in terms of the chordal distance, given by $d_{\min }=\sqrt{1-\left|\overline{\mathbf{f}}_{k}^{H} \overline{\mathbf{h}}_{k}\right|^{2}}$, for the proposed double-cap DCB, the single polar cap DCB with 4 bit and RVQ codebooks with 4, 5 and 6 bits feedback. Even with 6 bit feedback, the RVQ codebook gives larger quantization errors than the double-cap DCB with 4 bit feedback. Also, the double-cap DCB produces small quantization errors as compared to the single polar cap DCB, implying that the double-cap DCB tracks the channel more effectively. It is seen that the opposite scaling (i.e. scaling up the DCB when inner cap codeword is selected and vice versa) results in a poor quantization performance compared to other DCBs.

\section{CONCLUSION}

We have proposed an effective double-cap DCB design that is based on both spherical and polar cap structures. While most

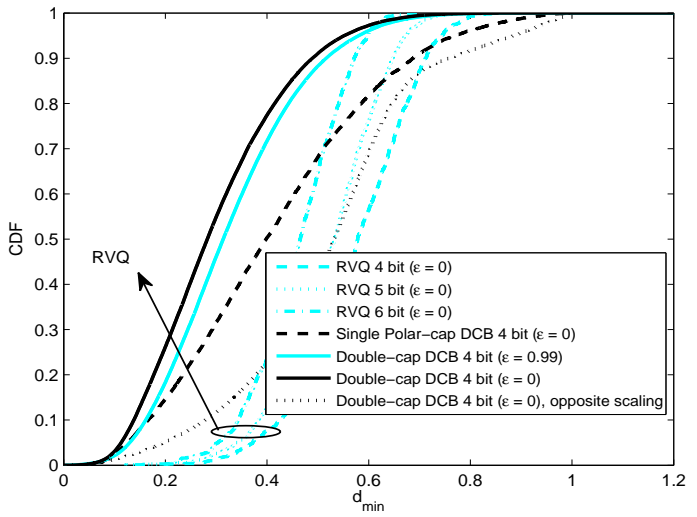

Fig. 4. $\mathrm{CDF}$ of quantization errors at $\mathrm{v}=3 \mathrm{~km} / \mathrm{h}$ (100 feedback intervals).

of the existing work assumes that the BS has perfect temporal correlation or spatial correlation knowledge, the key to the proposed method is to leverage the information inherent in a two cap structure to enable scaling up and scaling down the cap radii when necessary. Even without correlation knowledge at the BS, numerical results show the effectiveness of the double-cap DCB as compared to the single polar cap DCB.

\section{REFERENCES}

[1] D. Love, R. W. Heath Jr., and T. Strohmer, "Grassmannian beamforming for multiple-input multiple-output wireless systems," IEEE Trans. on Inf. Theory, vol. 49, no. 10, pp. 2735 - 2747, 2003.

[2] C. Au-Yeung and D. Love, "On the performance of random vector quantization limited feedback beamforming in a MISO system," IEEE Trans. Wireless Commun., vol. 6, no. 2, pp. 458 - 462, 2007.

[3] D. J. Love and R. W. Heath Jr, "Limited feedback diversity techniques for correlated channels," IEEE Trans. Veh. Tech., vol. 55, no. 2, pp. 718 $-722,2006$.

[4] V. Raghavan, R. W. Heath Jr., M. Sayeed et al., "Systematic codebook designs for quantized beamforming in correlated MIMO channels," IEEE J. Sel. Areas Commun., vol. 25, no. 7, pp. 1298 - 1310, 2007.

[5] T. Kim, D. Love, and B. Clerckx, "MIMO systems with limited rate differential feedback in slowly varying channels," IEEE Trans. Commun., vol. 59, no. 4, pp. 1175 - 1189, 2011.

[6] J. Choi, B. Clerckx, N. Lee, and G. Kim, "A new design of polarcap differential codebook for temporally/spatially correlated MISO channels," IEEE Trans. Wireless Commun., vol. 11, no. 2, pp. 703 711, 2012.

[7] J. Mirza, P. Dmochowski, P. Smith, and M. Shafi, "Limited feedback multiuser MISO systems with differential codebooks in correlated channels," in Proc. IEEE Int. Conf. on Commun., June 2013.

[8] — "A differential codebook with adaptive scaling for limited feedback MU MISO systems," IEEE Wireless Commun. Lett., vol. 3, no. 1, pp. $2-5,2014$.

[9] T. Abe and G. Bauch, "Differential codebook MIMO precoding technique," in Proc. IEEE Global Telecommun. Conf., Nov. 2007.

[10] T. Inoue and R. W. Heath, "Geodesic prediction for limited feedback multiuser MIMO systems in temporally correlated channels," in Proc. IEEE Symposium on Radio and Wireless, Jan. 2009.

[11] K. Kim, I. H. Kim, and D. Love, "Utilizing temporal correlation in multiuser MIMO feedback," in Proc. 42nd Asilomar Conf. Signals, Syst. and Comput., Oct. 2008.

[12] IST-WINNER II, "Deliverable 1.1.2 v.1.2, WINNER II channel models, IST-WINNER2," (http://projects.celticinitiative.org/winner+/deliverables.html), Tech. Rep., 2007.

[13] M. Sadek, A. Tarighat, and A. Sayed, "A leakage-based precoding scheme for downlink multi-user MIMO channels," IEEE Trans. Wireless Commun., vol. 6, no. 5, pp. 1711 - 1721, 2007.

[14] V. Raghavan, S. V. Hanly, and V. V. Veeravalli, "Statistical beamforming on the grassmann manifold for the two-user broadcast channel," IEEE Trans. Inf. Theory, vol. 59, no. 10, pp. 6464 - 6489, 2013.

[15] N. Jindal, "MIMO broadcast channels with finite-rate feedback," IEEE Trans. Inf. Theory, vol. 52, no. 11, pp. 5045 - 5060, 2006. 